\title{
Thermal Management with Solid-Fluid Slip Irreversibility Treatment in Conjugate Microdevices
}

\author{
E. O. B. Ogedengbe \\ Product Modelling \& Design Department, Energhx Consulting, 90 Woodridge Crescent, Suite 401, Nepean, ON, Canada K2B 7T1 \\ Correspondence should be addressed to E. O. B. Ogedengbe, ogedengbe@energhx.com
}

Received 15 July 2008; Accepted 9 January 2009

Recommended by Kimon A. Antonopoulos

\begin{abstract}
A numerical study of the effect of slip flow irreversibility and axial conduction in microdevices with a conjugate heat transfer between unmixed streams is presented. The effects of axial conduction due to parallel flows for thermal management in energy systems are investigated. Silicon substrate containing rectangular microchannels is simulated using a finite volume, staggered coupling of the pressure-velocity fields. The entropy generation transport within the entire system is analyzed and coupled with the solution procedure. The effects of channel size perturbation, Reynolds number, and pressure ratios on the thermal performance and exergy destruction are presented. Comparative analysis of the axial conduction and flow irreversibility between parallel flow on thermal management is studied. A proton exchange membrane (PEM) fuel cell model is used as a quality indicator to access the importance of the exergy-based design method.
\end{abstract}

Copyright (๑) 2009 E. O. B. Ogedengbe. This is an open access article distributed under the Creative Commons Attribution License, which permits unrestricted use, distribution, and reproduction in any medium, provided the original work is properly cited.

\section{Introduction}

Increasing demand for performance of MEMS, including microelectronic devices and microfuel cells, has often overweighed recent advances in the development of the very large-scale integration (VLSI) technology. These advances, particularly in electronic industry, require increase in circuit density and operating speed which generates more heat than the level of development of microheat sink can handle. Also, the design of innovative microfuel cell technologies has driven the ongoing investigations into efficient energy conversion at the near wall microchannels [1]. Apart from the problem of water flooding at the cathode side of the PEM fuel cell, inadequate analysis of the exchange of energies at the near wall of the enclosing channels constitutes unresolved thermal mismanagement. It is anticipated that future generation of microelectronic devices and microprocessors will require heat sink capacity in excess of $1000 \mathrm{~W} / \mathrm{cm}^{2}$ [2].

Parallel microchannels are utilized in the design configuration of fluid flows in microfuel cells and microheat sinks for electronic packages. This configuration has been proposed as effective and promising cooling techniques for microelectronic devices [3]. Consequently, increasing attention has been drawn to the study of fluid flow and heat transfer characteristics in a single microchannel [46]. Reducing exergy destruction of fluid motion through microchannels can have beneficial impact on reducing pressure losses and input power needed for these flow control methods. Although these effects are insignificant in hightemperature applications like solid electrolytes fuel cells and compact heat exchangers, where effective heat recoveries are cogent on microscale treatment of environmental barrier coatings, irreversibility treatments in microdevices can provide efficient design of all constituent energy systems. Previous studies by Ogedengbe et al. [6] have identified the importance of pressure ratios, channel size perturbation, and entropy production in optimal design of microfluidic devices. Their studies focused on flow characteristics with irreversibility effects in a single microchannel. Maxwell's first-order boundary conditions were imposed explicitly at the wall of the microchannel. While the interactions of kinetic and thermal energies at the near-wall were analyzed, the effect of the second component of the wall-slip, based on axial thermal gradient, was not investigated since the problem setup involves a single channel flow.

This paper develops a finite volume model of developing thermal boundary layer on conjugate flow model, using EnerghxFlow solver due to the effect of heat conduction from 


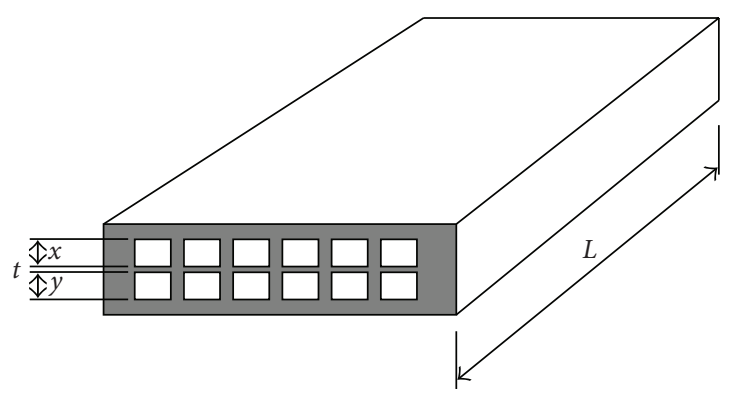

$x$ Height of primary microchannel

$y$ Height of secondary microchannel

$t$ Thickness of the interconnection core

$L$ Length of the microchannel

FIgURE 1: Schematic of conjugate microchannel system.

TABle 1: Model thermophysical properties.

\begin{tabular}{lc}
\hline Flow parameters & Value \\
\hline Length of microchannels $(\mu \mathrm{m})$ & 2560 \\
Square size of microchannels $(\mu \mathrm{m})$ & 1.0 \\
Dynamic viscosity of gas $\left(\mathrm{Ns} / \mathrm{m}^{2}\right)$ & 0.0000164 \\
Density of gas $\left(\mathrm{kg} / \mathrm{m}^{3}\right)$ & 1.2498 \\
Density of solid $\left(\mathrm{kg} / \mathrm{m}^{3}\right)$ & 998.2 \\
Specific gas constant of gas $(\mathrm{J} / \mathrm{Kg} \mathrm{K})$ & 296.8 \\
Specific gas constant of solid $(\mathrm{J} / \mathrm{Kg} \mathrm{K})$ & $390+0.9 T$ \\
Outlet pressure $(\mathrm{Pa})$ & 100800.0 \\
Pressure ratio, $P_{\text {in }} / P_{\text {out }}$ & $1.34,3.00$ \\
\hline
\end{tabular}

the solid wall between microdevices. Analysis on how both pressure differences and axial thermal conduction affect the slip flow irreversibility and exergy losses in microchannels will be presented. Second law analysis will be performed and entropy generation will be determined for fluid motion in microchannels. Results will be presented and discussed for parallel microchannel arrangement (see Figure 1).

\section{Thermophysical Model}

Figure 1 represents the schematic of conjugate microchannel system, comprising the flow of primary and secondary fluid streams in parallel. For a fuel cell system, the fuel flows through the primary channels while the air flows through the secondary channel streams. But, in a microheat sink system, the primary and secondary channels carry the cold and hot fluid streams, respectively. The schematic of the microheat sink is investigated in this paper, where the size falls within the regime of flow with slip boundary condition (see Table 1). Three-dimensional effect is neglected as symmetry assumption is reasonable with the scale of the flow field. Both the hot and cold streams, as shown in Figure 2, are modeled using the thermophysical properties of nitrogen. The solid material is modeled with the thermophysical properties of silicon. Capillarity, interface, microconvection, and other transport effects through the interconnection or porous material require microscale modeling which cannot be

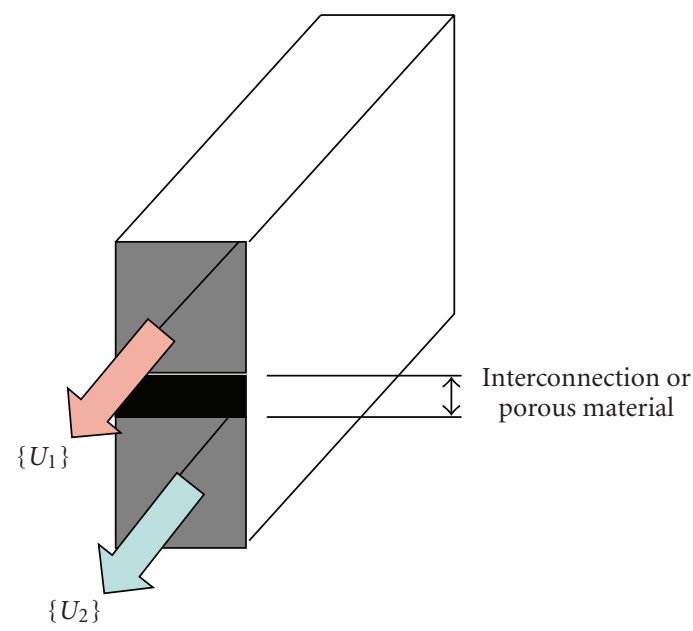

FIGURE 2: Flow arrangement in parallel microchannels.

captured under continuum assumption. A hybrid numerical scheme based on both continuum and lattice Boltzmann (for simulation of flows in porous medium) assumptions can be developed, for relevant investigation of previously neglected model kinetics, that are capable of improving the efficiency heat recovery energy systems, especially processes involving phase change and crystal growth [7].

One major controversy with slip flow condition at the near wall in microchannels (when the Knudsen number is between 0.001 and 0.1 ) is that the mechanism of exchange between kinetic and thermal energies is variedly treated. Since entropy production encompasses both friction and thermal irreversibilities, it provides key insight to enhance heat exchange without sacrificing excessive pressure losses that contribute to higher pumping power.

In addition to convincingly evidence recently published on this issue [6], this paper introduces the effect of axial conduction due to exchange of thermal energy across the separating wall of parallel microchannels.

\section{Coupled Heat-Mass Model}

The computational domain encompasses both the siliconmodeled solid and two-unmixed stream of gas flowing in parallel direction. The steady-state governing equations represent the continuity equations, momentum equations, energy equations for both solid and gas streams. The equations for the gas streams (where subscript $i=1$ or 2 and represents upper hot stream or lower cold stream) are as follows:

$$
\begin{gathered}
\frac{\partial\left(\rho_{i}\right)}{\partial t}+\nabla \cdot\left(\rho_{i} u_{i}\right)=0, \\
\frac{\partial\left(\rho_{i} u_{i}\right)}{\partial t}+\nabla \cdot\left(\rho_{i} v_{i} u_{i}\right)=-\frac{\partial p_{i}}{\partial x}+\nabla \cdot\left(\mu_{i} \nabla u_{i}\right), \\
\frac{\partial\left(\rho_{i} v_{i}\right)}{\partial t}+\nabla \cdot\left(\rho_{i} v_{i} v_{i}\right)=-\frac{\partial p_{i}}{\partial y}+\nabla \cdot\left(\mu_{i} \nabla v_{i}\right), \\
\frac{\partial\left(\rho_{i} c_{P i}\right)}{\partial t}+\nabla \cdot\left(u_{i} T_{i}\right)=\nabla \cdot\left(k_{i} \nabla T_{i}\right) .
\end{gathered}
$$




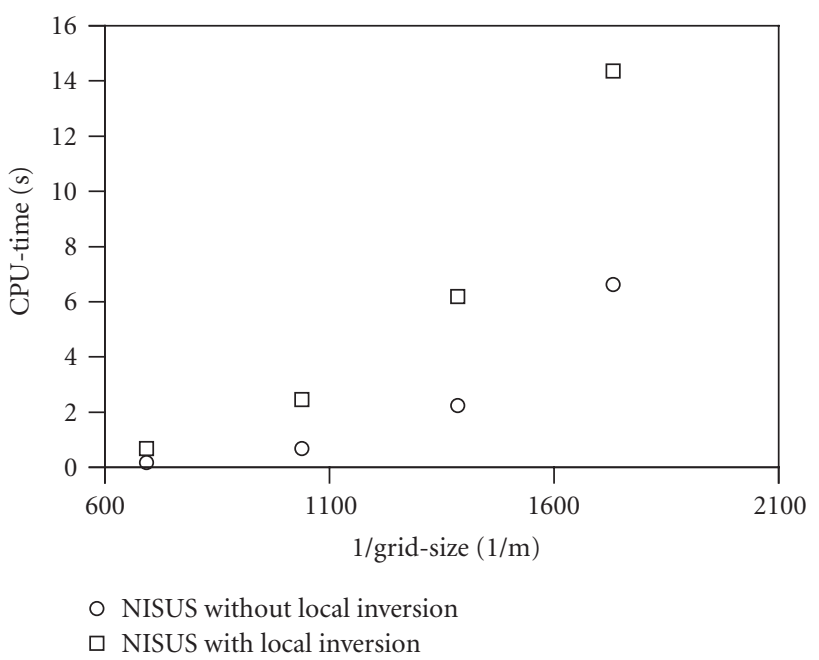

FIgURE 3: Computational time saving advantage of NISUS.

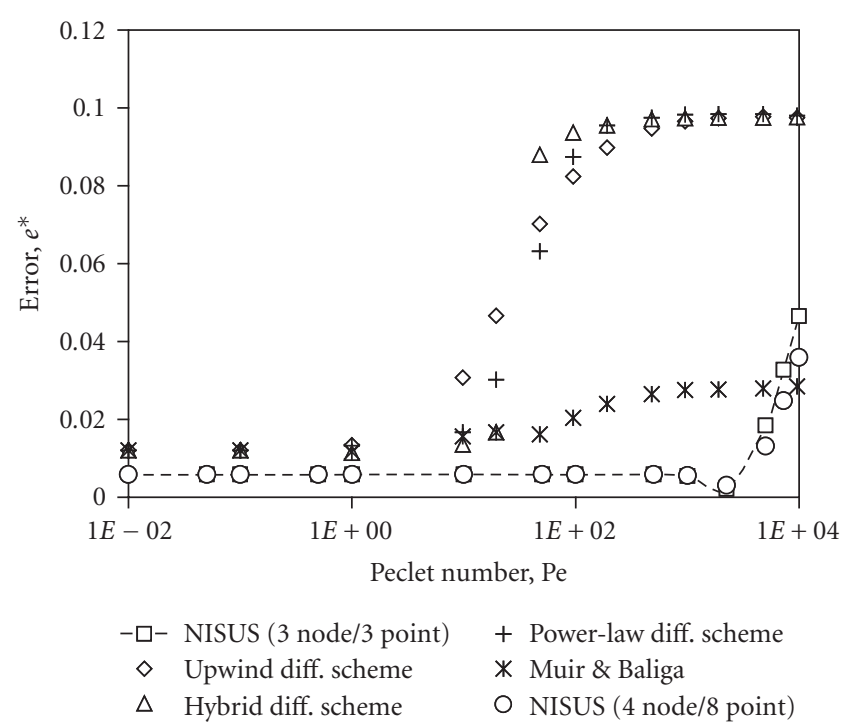

FIGURE 4: Accuracy of NISUS over previous advection schemes.

where $a, \phi$, and $b$ refer to the finite volume coefficient, scalar variable (such as the nodal velocity in the $u$-momentum equation), and the source term, respectively. Detailed formulation of the finite volume-based NISUS is documented elsewhere [4]. Figures 3 and 4 present the computational time-saving advantage and accuracy, respectively, of NISUS over other advection schemes.

The inversion of a local $12 \times 12$ matrix (for a 3D hexahedral control volume element) refers to the upwind coefficient matrix that needs to be inverted in order to represent the integration point variables in terms of nodal variables alone. Significantly, the time-gain pattern presents an increasing propagation with further refinement and larger problem size. In term of accuracy, the predicted results are compared against other schemes (i.e., upwind, hybrid, power-law, and Muir-Baliga) between the range $0<$ $\mathrm{Pe} \leq 10^{4}$. The numerical error of the proposed scheme is maintained at an average of about 0.0058 between $0<\mathrm{Pe} \leq$ $10^{3}$, while showing excellent accuracy and numerical stability in comparison with other schemes. It should be noted that the test case (i.e., radial heat in a rotating hollow spheres) involves flows with recirculation and has been demonstrated as applicable to the design of a radial lubrication systems [11].

The modeling of the heat-mass equations governing the energy system, as described above, has not included the quality of the available quantities of energies. The design of energy systems without consideration of the transport of entropy is based on the First law of thermodynamics, while the second law analysis only guarantees a quality-based design. Section 4 presents the exergetic considerations and analysis of conjugate microdevices.

\section{Exergetic System Analysis}

The second law equation describes the state of irreversibility within the boundaries of energy systems. Due consideration

$$
a_{P} \phi_{P}=\sum a_{n b} \phi_{n b}+b,
$$

are imposed at the solid-fluid interface. All other wall The numerical treatments of advection terms in (2)(4) represent a significant consideration in the design of energy systems, since these efforts control the product design (he non-inverted skew upwind scheme (NISUS) discretization of NISUS introduces the concept of non inverted computation of convected variables into the SIMLEC formulation. Equations (1)-(5), after integration with volume boundary which becomes the sums of integrated conservation balance can be simplified with the following standard summation form: 
of this law in addition to the first law can provide better estimate of the quality of available heat energy recoverable via the serving stream of the conjugate micro devices. These two laws of thermodynamics can be written as [12]

$$
\begin{aligned}
& \frac{d E}{d t}=\dot{Q}_{O}+\sum_{i=l}^{n} \dot{Q}_{i}-\dot{W}+\sum_{\text {in }} \dot{m} h_{t}+\sum_{\text {out }} \dot{m} h_{t}, \\
& \dot{S}_{\text {gen }}=\frac{d S}{d t}-\frac{\dot{Q}_{O}}{T_{O}}-\sum_{i=l}^{n} \frac{\dot{Q}_{i}}{T_{i}}-\dot{W}+\sum_{\text {in }} \dot{m} h_{t}+\sum_{\text {out }} \dot{m} h_{t} .
\end{aligned}
$$

By eliminating $\dot{Q}_{O}$ from (9), the work rate output can be maximized as

$$
\begin{aligned}
\dot{W}= & -\frac{d}{d t}\left[E-T_{O} S\right]+\sum_{i=l}^{n}\left(1-\frac{T_{O}}{T_{i}}\right) \dot{Q}_{i}+\sum_{\text {in }} \dot{m}\left(h_{t}-T_{O} s\right) \\
& -\sum_{\text {out }} \dot{m}\left(h_{t}-T_{O} s\right)-T_{O} \dot{S}_{\text {gen }} .
\end{aligned}
$$

Since $\dot{S}_{\text {gen }}$ cannot be negative, the maximum possible work from the system is obtained at the minimum value of $T_{O} \dot{S}_{\text {gen }}$, known as the lost available work or Gouy-Stodola theorem. In order to understand the application of this theorem to conjugate heat transfer design, it will be useful to comprehend the process of entropy generation via the interaction of the streams with the walls.

System optimization demands exergy analysis for all energy systems, where power or refrigeration effect is operational. In this case and as it applies to heat transfer design, the first law which deals with the conservation of energy will not be adequate in order to capture the heat and work interaction through the conjugate system.

From the first law,

$$
\dot{m} d h=q^{\prime} d x
$$

Moreover, assuming steady-state condition with no work and heat loss or gain from the environment, the second law states that

$$
d \dot{S}_{\text {gen }}=\frac{d \dot{Q}}{T \pm \nabla T}+\dot{m} d s \geq 0, \quad \text { for each side }
$$

while the \pm sign denotes either the hot or cold stream of the heat exchanger. Now, the canonical entropy relationship states that

$$
\frac{d h}{d x}=T \frac{d s}{d x}+\frac{1}{\rho} \frac{d p}{d x} .
$$

Therefore, entropy generation term (after linking (11)-(13)) is

$$
\dot{S}_{\text {gen }}^{\prime}=\frac{d S_{\text {gen }}}{d x}=\frac{q^{\prime} \Delta T}{T^{2}(1+\tau)}+\frac{\dot{m}}{\rho T}\left(-\frac{d p}{d x}\right),
$$

where $\tau=\Delta T / T$, the dimensionless temperature difference. This equation reveals the two basic components of entropy generation, including the temperature gradient term and the pressure gradient term. Since the heat transfer gradient is directly proportional to the temperature gradient, it implies that the entropy generation rate for the thermal component is proportional to the square of the dimensionless temperature difference $\tau$, and this term plays a vital role in the minimization of the generation of entropy within the energy system. The importance of entropy generation to the design of energy systems can be significant even with microdevices, especially by incorporating the thermal kinetic energy influence of slip at the near wall at microscale.

The balance of entropy for a control volume can be written as [6]

$$
\frac{\partial(\rho s)}{\partial t}+\nabla \cdot(\rho v s)=-\nabla \cdot\left(\frac{q}{T}\right)+\dot{S}_{\mathrm{gen}}^{\prime},
$$

where $\dot{S}_{\text {gen }}^{\prime}$ refers to the entropy production rate (per unit volume). When this entropy production rate is multiplied by a suitable reference temperature, it represents the local rate of exergy destruction, $\dot{X}_{d}^{\prime \prime \prime}$, per unit volume. Using the Gibbs equation, it can be shown that the rate of exergy destruction for microchannel flows can be written directly in terms of both the velocity and temperature gradients as follows:

$$
\begin{aligned}
\dot{X}_{d}^{\prime \prime \prime}= & \mu\left(\frac{\partial u}{\partial y}+\frac{\partial v}{\partial x}\right)^{2}+2 \mu\left(\left(\frac{\partial u}{\partial x}\right)^{2}+\left(\frac{\partial v}{\partial y}\right)^{2}\right) \\
& +\mu\left(\left(\frac{\partial T}{\partial x}\right)^{2}+\left(\frac{\partial T}{\partial y}\right)^{2}\right) .
\end{aligned}
$$

Thus, wall slip in (6) affects the velocity and temperature profiles and resulting exergy destruction in (16). This frictional dissipation of kinetic energy leads to pressure losses in microchannels, which depend on thermal convection and streamwise temperature gradients outlined in (6).

A finite volume method was developed based on conjugate conduction of thermal energy across the wall boundary separating the two fluid streams. By integrating each conservation equation over the discrete control volume, the algebraic equations are obtained in terms of nodal variables. An iterative solver was used to solve these resulting algebraic equations.

Using the resulting velocity field solutions, exergy losses are calculated based on (16). Performing spatial differencing of (16), the exergy destruction rate per unit volume at node $P$ becomes

$$
\begin{aligned}
\dot{X}_{d}^{\prime \prime \prime}= & \mu\left(\frac{u_{N}-u_{S}}{\Delta y}+\frac{v_{E}-v_{W}}{\Delta x}\right)^{2} \\
& +2 \mu\left(\left(\frac{u_{E}-u_{W}}{\Delta x}\right)^{2}+\left(\frac{v_{N}-v_{S}}{\Delta y}\right)^{2}\right) \\
& +\mu\left(\left(\frac{T_{E}-T_{W}}{\Delta x}\right)^{2}+\left(\frac{T_{N}-T_{S}}{\Delta y}\right)^{2}\right),
\end{aligned}
$$

where $\Delta x$ and $\Delta y$ refer to the grid spacing in the $x$ and $y$ directions, respectively. All terms in (17) remain positive since they are sums of squared terms. Physically, mechanical energy is irreversibly dissipated to internal energy through fluid friction, which produces entropy 


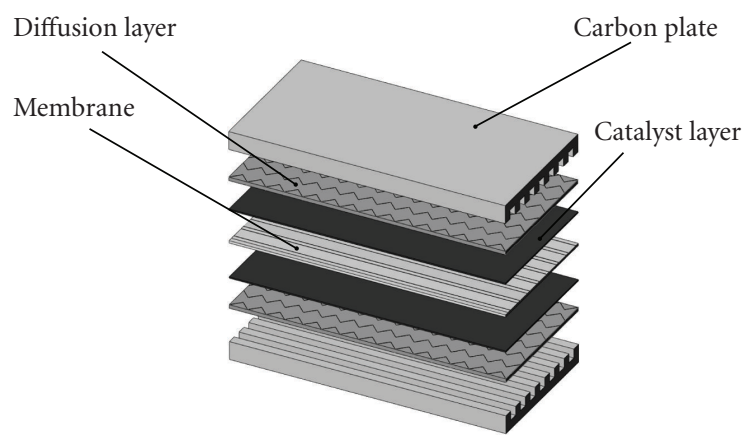

Figure 5: Exploded view of PEMFC's MEA.

and requires additional input power to overcome pressure losses through the microchannel. Reducing entropy production from thermofluid irreversibilities in microfluidic devices can provide useful benefits like improving the efficiency of energy utilization within the overall system and pinpointing the locations, causes and types of thermodynamic losses. In Section 5, numerical results from microchannel flow simulations will be presented and discussed.

\section{Thermal Management in PEM Fuel Cell}

The proton exchange membrane fuel cell (PEMFC) is an alternative energy-conversion device with the potential for significantly increased energy density [13]. While the problem of waste-water management in PEMFCs is receiving increasing attention, thermal management creates a destructive load in the design of portable power systems. It has been predicted that the rate of heat removal will become a critical parameter in the operation of fuel cells [14]. The PEMFC schematic shown in Figure 5 includes the heat generation from the catalyst layer at the cathode of the PEMFC. The interconnection material represents the lumped model for the material assembly, including the graphite plate, gas channels, electrodes, and the membrane. Detailed modeling of the transfer of heat across this material involves the derivation of the effective thermal conductivity and inclusion of the heat generation term in the energy equation. The half reaction at the anode releases both protons, which conduct through the membrane, and electrons which are transferred to the cathode through an external circuit. The complete reaction of both protons and electrons with oxygen (from air) in the catalyst layer of the cathode is exothermic, representing the source of the destructive heat load.

The control of the heat generated from the exothermic reaction at the cathode of the membrane electrolyte assembly (MEA) is significant to the performance of PEMFCs. Cooling plate with microchannels adopted recently for the purpose of regulating the flow of water vapor at the MEA; as both water vapor starvation and water-flooding reduce the output current density of fuel cells. Detail study of the design of cooling plate for thermal management in PEMFCs is documented elsewhere [15].

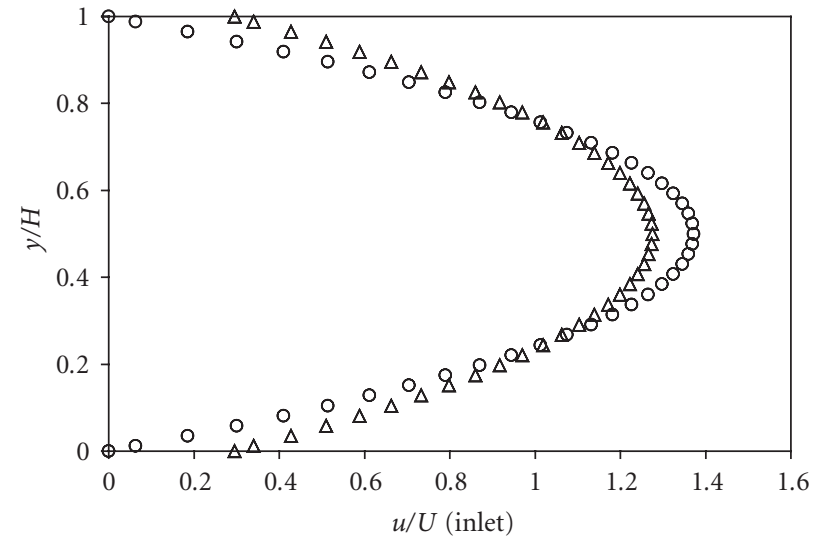

$\Delta$ NISUS predicted slip flow

○ No-slip analytical solution $P_{\text {out }} / P_{\text {in }}=1.34$

FIGURE 6: Velocity profile validation with slip boundary conditions.

\section{Results and Discussion}

Numerical predictions of nitrogen gas flow in microchannels are presented in this section. Numerical simulations for the primary (hot) gas stream with various grid configurations are studied and grid-independent results are presented in this section. Figure 6 validated the predicted velocity profile within a channel by comparing results with analytical results. A slip scale of about 0.3 is observed at the pressure ratio of 1.34 and the profile agrees with analytical results, showing error of about $1 \%$ at the near wall of the channel. The reduction of the maximum velocity for slip profile appears to be due to the thermal kinetic energy exchange at the boundary of the microchannels. Slip irreversibility effects also include the demand of computational resources at the region of flow, where the greatest gradients of flow variables are experienced, as shown in Figure 7.

The axial conduction temperature gradient has an influence on the slip boundary condition. However, it can be shown that entropy production from fluid friction depends on both velocity and temperature gradients within the flow field. Figure 8 predicted the effect of the axial conduction of thermal variable and the momentum accommodation coefficient along the length of the microdevice. According to (6), the wall slip velocity is dependent on both the momentum accommodation coefficient and the axial temperature gradient. This dependence directly impacts friction with an unusual thermal mechanism of entropy production in micro- and nanoscale channels. This phenomenon may relate to previous studies by Wang et al. [9], which indicate that thermal energy may be temporarily converted to kinetic motion in a cohesive manner within very small scale systems. It, therefore, implies that wall temperature gradients affect entropy production in the fluid stream. Efforts to fully comprehend this mechanism physically require a statistically based balance of momentum and energy equations for intermolecular motion near the wall. As channel sizes diminishes to micro- and nanoscales, the number of molecule interactions within the channel decreases. 


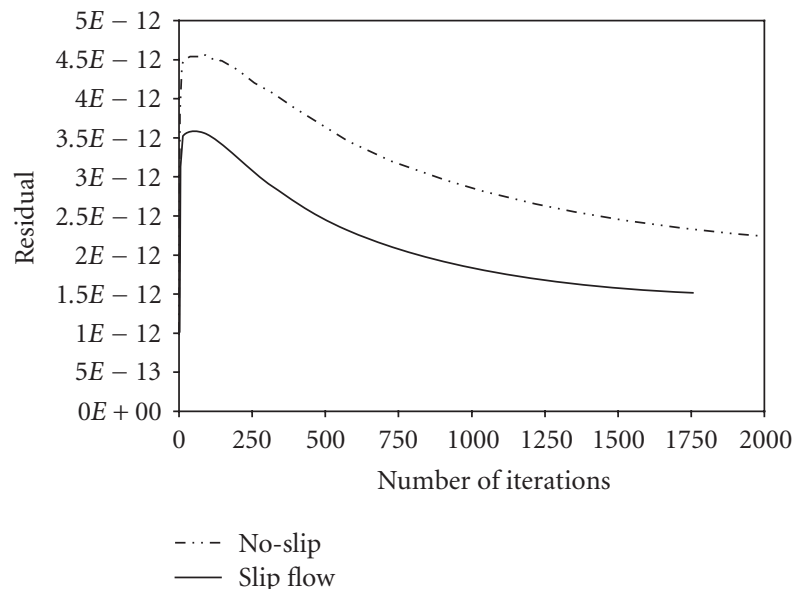

FIGURE 7: Computational effects of slip irreversibility.

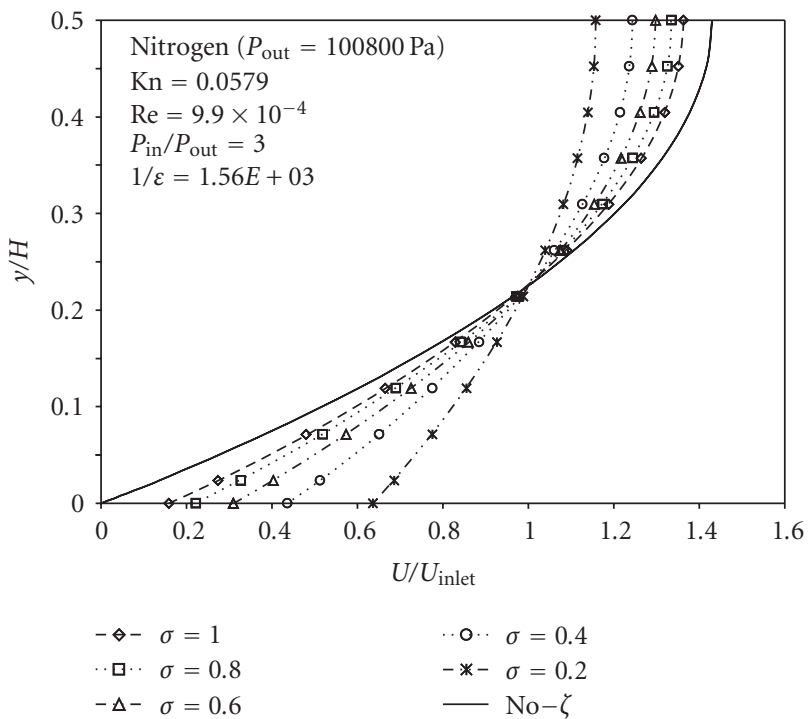

FIGURE 8: Effect of axial conduction and momentum accommodation coefficient.

Interaction between gas and solid phase molecules can influence the extent of energy transfer and accommodation in gas-surface collisions at micro- or nanoscales. The likelihood of random molecular motions within the wall (in the form of internal energy) being aligned in the direction of reflected motion of impacting fluid molecules rises when the channel size diminishes. In other words, when fewer wall molecules are considered in smaller channels, their alignment toward the gas velocity vector may contribute an effective temporary rise of kinetic energy. In this way, local temperature gradients affect the wall slip in (6) and subsequently entropy production in the fluid stream, when formulating the second law. Thermal energy exchange affects the wall slip, thereby potentially reducing frictional entropy production. This has important implications for operation and efficiency of microfluidic devices.

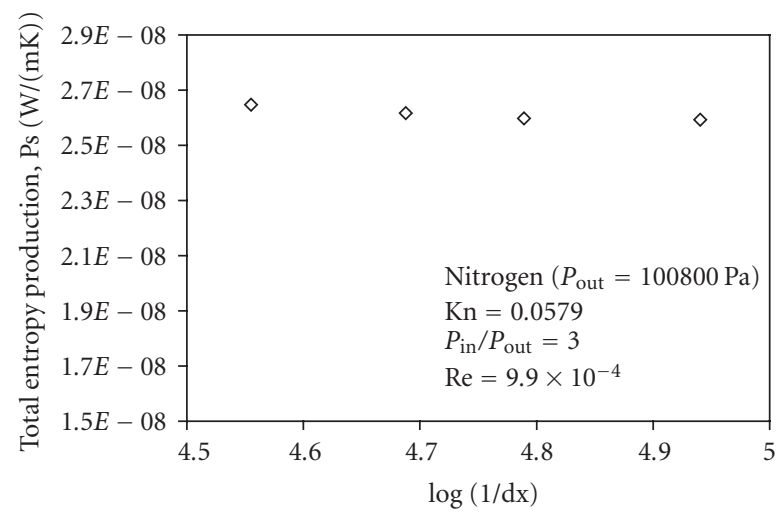

FIGURE 9: Grid independency based on entropy production.

Figure 9 shows the effects of grid refinement on the predicted entropy production. The entropy production remains nearly constant over a range of grid spacings, which suggests that the results are essentially grid independent.

\section{Conclusions}

Near-wall irreversibility effect on the solid-fluid interface of conjugate microdevice has been analyzed. Exergetic design procedure of microfluidic devices based on second law was coupled with the Navier-Stokes model, developed and packaged as a robust EnerghxFlow solver. The present model is capable of simulating slip irreversibility effects toward the design of a microheat sink. However, and especially for a microfuel cell and other devices with porous interconnection, a complete treatment of microscale phenomena like microconvection within the solid-fluid interface will require the development of hybrid formulation based on both continuum assumption and Lattice-Boltzmann technique. Sensitivity analysis of the effect of the momentum accommodation coefficient and the axial conduction due to the conjugate transfer of heat across the solid-fluid interface has been presented. The magnitude of the momentum accommodation coefficient is inversely proportional to the wall-slip velocity and plays a significant role in the dynamics of the thermal kinetic exchange at the near wall.

\section{Nomenclatures}

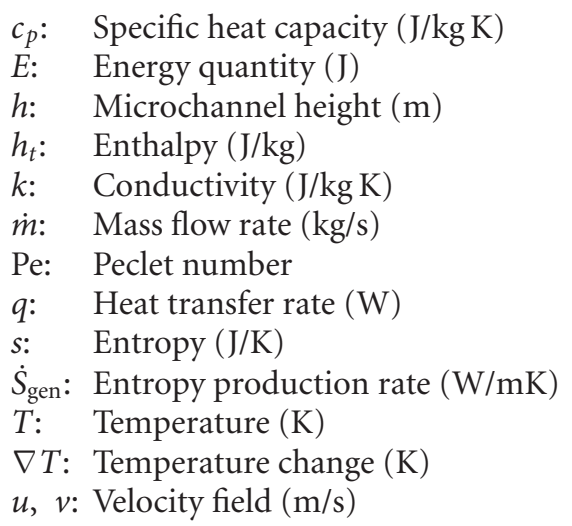


$\{U\}:$ Field variables

$\dot{W}: \quad$ Work rate $(\mathrm{J} / \mathrm{s})$

$\dot{X}_{d}^{\prime \prime \prime}: \quad$ Exergy destruction (per unit volume)

$\xi_{1}, \xi_{2}:$ Slip coefficients

$\lambda$ : $\quad$ Mean-free path

$\mu$ : $\quad$ Viscosity of the fluid (Pas)

$\rho: \quad$ Density of the fluid $\left(\mathrm{kg} / \mathrm{m}^{3}\right)$

$\sigma: \quad$ Momentum accommodation coefficient

$\tau: \quad$ Dimensionless temperature difference.

\section{Acknowledgments}

The efforts of the staff of Energhx Consulting with data reporting and documentation are appreciated. Also, the author is grateful to Dare A. Adetan, Greg F. Naterer, and Marc A. Rosen for their useful contributions. Financial support by the Natural Sciences and Engineering Research Council of Canada is gratefully acknowledged.

\section{References}

[1] E. O. B. Ogedengbe, G. F. Naterer, and M. A. Rosen, "Convective exergy losses of developing slip flow in microchannels," International Journal of Exergy, vol. 4, no. 4, pp. 384-400, 2007.

[2] J. Darabi, M. M. Ohadi, and D. DeVoe, "An electrohydrodynamic polarization micropump for electronic cooling," Journal of Microelectromechanical Systems, vol. 10, no. 1, pp. 98-106, 2001.

[3] D. B. Tuckerman and R. F. W. Pease, "High-performance heat sinking for VLSI," IEEE Electron Device Letters, vol. 2, no. 5, pp. 126-129, 1981.

[4] E. O. B. Ogedengbe, G. F. Naterer, and M. A. Rosen, "Slipflow irreversibility of dissipative kinetic and internal energy exchange in microchannels," Journal of Micromechanics and Microengineering, vol. 16, no. 10, pp. 2167-2176, 2006.

[5] E. O. B. Ogedengbe, G. F. Naterer, and M. A. Rosen, "Dissipative kinetic and thermal energy exchange in microchannel flows," in Proceedings of the Canadian Society for Mechanical Engineering Forum (CSME '06), pp. 1-10, Kananaskis, Canada, May 2006, Section WB2: Experimental Fluid Mechanics 2, paper 4.

[6] E. O. B. Ogedengbe, G. F. Naterer, and M. A. Rosen, "Finite volume dielectrophoretic model of slip flow irreversibilities in microchannels," in Proceedings of the 15th Annual Conference of the CFD Society of Canada, Toronto, Canada, May 2007.

[7] E. Semma, M. El Ganaoui, R. Bennacer, and A. A. Mohamad, "Investigation of flows in solidification by using the lattice Boltzmann method," International Journal of Thermal Sciences, vol. 47, no. 3, pp. 201-208, 2008.

[8] J. C. Maxwell, "On stresses in rarefied gases arising from inequalities of temperature," Philosophical Transactions of the Royal Society of London, vol. 170, pp. 231-256, 1879.

[9] G. M. Wang, E. M. Sevick, E. Mittag, D. J. Searles, and D. J. Evans, "Experimental demonstration of violations of the second law of thermodynamics for small systems and short time scales," Physical Review Letters, vol. 89, no. 5, Article ID 050601, 4 pages, 2002.

[10] E. O. B. Ogedengbe, Non-inverted skew upwind scheme for numerical heat transfer and fluid flow simulations, Ph.D. thesis, University of Manitoba, Winnipeg, Canada, 2006.
[11] E. O. B. Ogedengbe and G. F. Naterer, "Reduced design time with NISUS convection modeling: application to a rotating lubrication system," in Proceedings of the 7th AfricaUSA International Conference of Manufacturing Technology (MANUTECH '04), Port Harcourt, Nigeria, July 2004.

[12] J. E. Hesselgreaves, Compact Heat Exchangers: Selection, Design and Operation, Pergamon, Amsterdam, The Netherlands, 2001.

[13] D. G. Strickland, S. Litster, and J. G. Santiago, "Current distribution in polymer electrolyte membrane fuel cell with active water management," Journal of Power Sources, vol. 174, no. 1, pp. 272-281, 2007.

[14] T. F. Fuller and J. Newman, "Water and thermal management in solid-polymer-electrolyte fuel cells," Journal of the Electrochemical Society, vol. 140, no. 5, pp. 1218-1225, 1993.

[15] E. O. B. Ogedengbe and M. A. Rosen, "Conjugate heat transfer with slip flow irreversibilities in PEM fuel cell microcooling," in Proceedings of the 2nd Climate Change Technology Conference (CCTC '09), Hamilton, Canda, May 2009. 

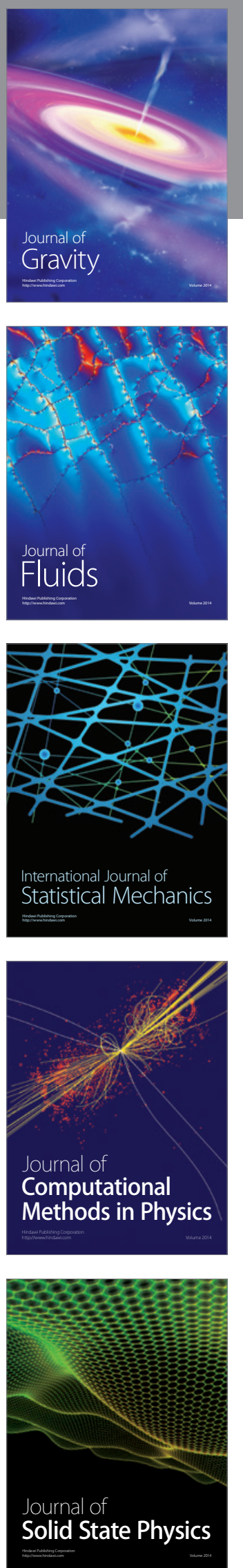

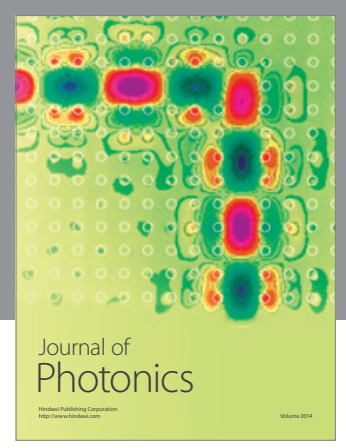

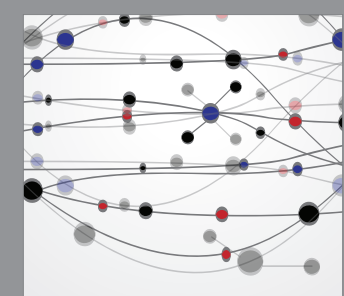

The Scientific World Journal
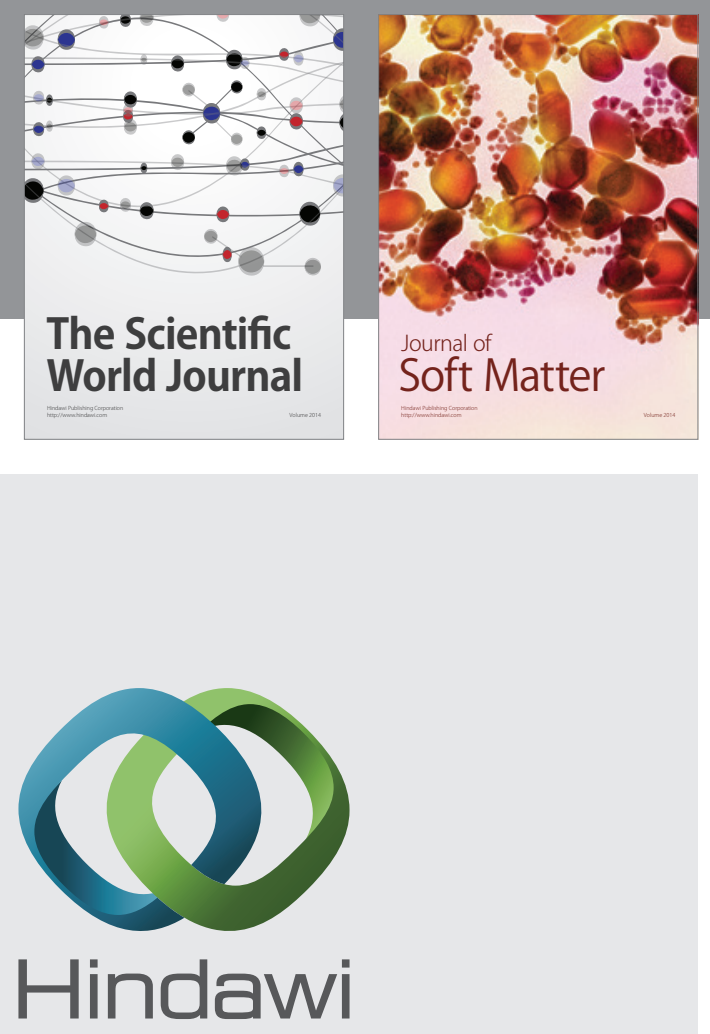

Submit your manuscripts at

http://www.hindawi.com
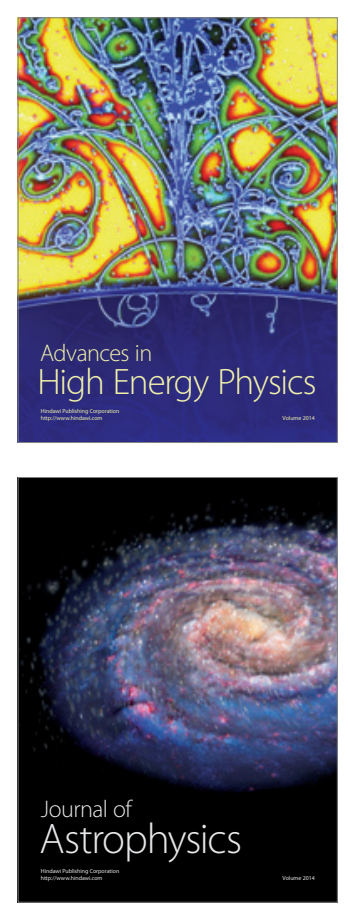
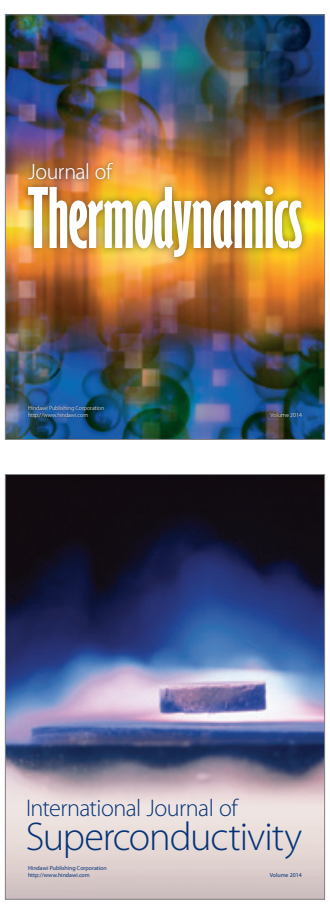
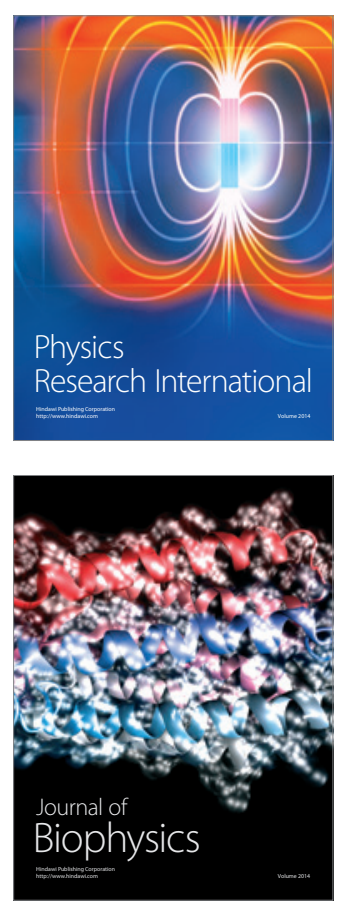
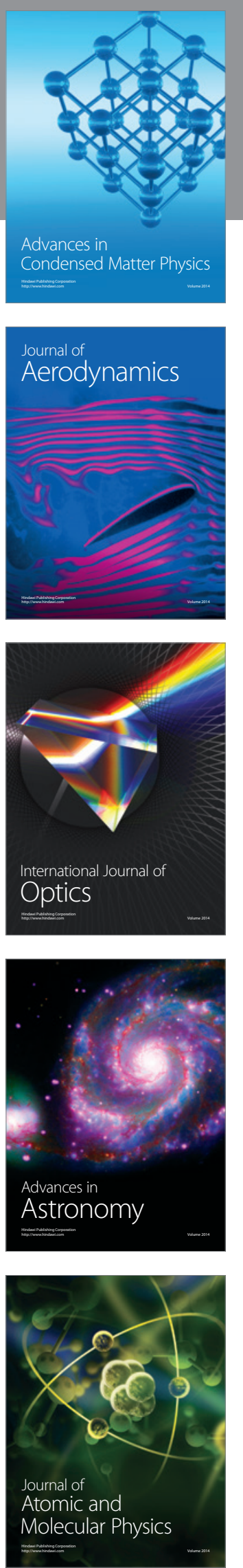